Argumentation Argumentation et Analyse du Discours

\title{
Analyse du Discours et analyse textuelle
}

Discourse analysis and text analysis

\section{Alain Lhomme}

\section{(2) OpenEdition}

Journals

Édition électronique

URL : http://journals.openedition.org/aad/3254

DOI : 10.4000/aad.3254

ISSN : 1565-8961

Éditeur

Université de Tel-Aviv

Référence électronique

Alain Lhomme, "Analyse du Discours et analyse textuelle ", Argumentation et Analyse du Discours [En ligne], 22 | 2019, mis en ligne le 15 avril 2019, consulté le 10 décembre 2020. URL : http:// journals.openedition.org/aad/3254; DOI : https://doi.org/10.4000/aad.3254

Ce document a été généré automatiquement le 10 décembre 2020.

\section{(c) $($ ) $(9)$}

Argumentation \& analyse du discours est mis à disposition selon les termes de la licence Creative Commons Attribution - Pas d'Utilisation Commerciale - Pas de Modification 4.0 International. 


\title{
Analyse du Discours et analyse textuelle
}

\author{
Discourse analysis and text analysis
}

\section{Alain Lhomme}

1 Formuler le projet d'une analyse du « discours » philosophique implique d'emblée une forme de déplacement, sinon de décentrement par rapport aux approches qui prévalent généralement chez les praticiens de l'analyse des «textes » philosophiques. Comme l'écrit Dominique Maingueneau,

l'attitude spontanée à l'égard des textes, en particulier des textes philosophiques, est de les considérer comme des textes, des réseaux de sens compacts et autonomes offerts à l'interprétation [...], mais les textes philosophiques, dès lors qu'ils sont des textes, impliquent des cadres qui leur donnent sens, et au premier chef la scène à travers laquelle ils s'énoncent (2015:65).

2 Cela revient à dire que, tout en reconnaissant le rôle essentiel que jouent les textes dans le champ littéraire et philosophique, l'Analyse du Discours refuse de les considérer selon une approche strictement "textualiste", dans la mesure où tout texte, en tant que texte, comporte une dimension énonciative où se joue une part essentielle de sa signification. Pour l'Analyse du Discours, «il ne s'agit pas seulement d'analyser des contenus, une organisation textuelle ou des procédés stylistiques, mais de rapporter les énoncés à des dispositifs de communication » (ibid. : 16). De la même manière, si l'Analyse du Discours s'estime fondée à « subvertir la distinction [...] entre “intérieur" et "extérieur" du texte » (17), c'est parce qu'elle privilégie le point de vue de l'énonciation, catégorie qui se situe en quelque sorte « entre » le texte et le contexte et qui «ne se laisse enfermer ni dans une problématique du texte, ni dans une problématique strictement sociologique » (16). Ce qui est plus particulièrement visé, c'est une "alternative ruineuse entre des approches d'ordre stylistique, qui sont censées appréhender le texte pour démonter ses rouages, et des approches d'ordre sociologique, qui dénoncent l'intériorité du texte comme illusoire et cherchent ses déterminations à l'extérieur, en particulier dans les institutions » (ibid.). 
3 Si donc les praticiens de l'Analyse du Discours entendent jeter un regard critique sur toute méthode qui, privilégiant l'immanence du texte, ignore la relation que celui-ci entretient nécessairement à sa propre extériorité, c'est parce que leur ambition est, à travers des notions comme celles d' "énonciation ", de "scène énonciative ", voire de "scénographie", de montrer que les textes, dans une large mesure, doivent être abordés comme des discours : non seulement ceux qui, conformément aux canons de la rhétorique classique, se désignent expressément comme tels ${ }^{1}$, mais tous ceux qui, par delà leur statut proprement textuel, prennent la forme accomplie du livre. C'est quand on passe en effet de la forme-texte à la forme-livre (en prenant soin, en particulier, d'y intégrer ce qu'on appelle le péritexte), que certaines dimensions trop souvent négligées par l'analyse textuelle viennent au premier plan: le texte-livre vise un auditoire, s'adresse à un destinataire, fait entendre une voix auctoriale, mobilise un dispositif souvent complexe d'énonciation. «Auditoire», «destinataire», «voix », "énonciation »: tous ces concepts ne peuvent apparemment trouver leur pleine application qu'au sein d'une conception qui rompt avec une approche purement «textualiste » des textes pour les considérer en même temps comme des discours.

4 L'ambition de l'Analyse du Discours est au demeurant bien plus ample : non seulement elle montre comment ces discours que sont aussi les textes produisent des effets dans le champ interdiscursif, mais elle met en évidence la façon dont ils participent d'un type déterminé d'institution discursive (qu'ils contribuent d'ailleurs souvent aussi à instituer), c'est-à-dire ouvrent sur un champ institutionnel, pratique, dont la prise en compte permet en retour d'éclairer certaines de leurs significations, voire de corriger les contresens que véhicule parfois une approche seulement textuelle ${ }^{2}$. C'est ici l'inspiration foucaldienne qu'il faut prendre en compte: les discours ont en tant que tels une fonction pratique, instituante.

5 Mais cette inspiration foucaldienne doit elle-même être tempérée : on sait que Foucault mettait un soin particulier à contourner l'analyse classique des doctrines et des systèmes. Son objet, ce sont les grandes «nappes de discours ». L'extension et le statut qu'il donne à l'événement discursif sont tels que la forme écrite se confond pour finir avec la forme archive, qu'il suffirait de lire pour en restituer la prolifération discursive, opposée au principe de raréfaction qui gouverne l'écriture des grands textes. On peut se demander dans ces conditions jusqu'à quel point cette référence foucaldienne ne devient pas quelque peu problématique lorsqu'il s'agit de s'intéresser à ces textes « hyperdenses » que livre la tradition philosophique classique.

6 Car le fait que, dans un texte, une pensée se trouve déposée, voire scellée, n'est pas un simple préjugé herméneutique ou le présupposé arbitraire d'un commentaire soucieux de reconduire la pensée à l'œuvre dans un texte à la «doctrine » ou au "système ». Dans le texte philosophique, une pensée se trouve élaborée et articulée. Le texte se présente donc aussi comme un véritable laboratoire de pensée: s'y formulent, s'y reprennent, s'y réélaborent des arguments et des concepts, et on peut se demander si, pour affronter cette autre dimension du textuel, l'Analyse du Discours est suffisamment armée. En un mot, l'accent mis légitimement par l'Analyse du Discours sur la dimension proprement discursive des textes philosophiques ne la conduit-elle pas à minorer d'autres dimensions du texte, où celui-ci se présente à l'inverse comme un objet irréductible à son alignement pur et simple sur la forme discours?

7 On mesure sans doute, au vu de ce simple propos introductif, l'enchevêtrement des questions que révèle la mise en parallèle et en concurrence de l'analyse textuelle et de 
l'Analyse du Discours. Pour commencer à démêler ces liens et ordonner un peu cette discussion, le plus expédient semble de commencer par décrire brièvement l'apport spécifique de l'Analyse du Discours dans l'étude des textes, relativement aux formes les plus classiques de l'analyse textuelle. D'exposer ensuite les dimensions du texte et/ou de la textualité que l'Analyse du Discours semble avoir quelque peine à prendre en compte. De montrer enfin quels autres types d'approche, autrement dit quelle forme d'analyse textuelle renouvelée serait requise pour faire droit à ces dimensions.

\section{L'intérêt d'une approche discursive des textes}

8 L'un des points sur lesquels insistent à juste titre les représentants de l'Analyse du Discours est que cette manière d'aborder les textes met fin à un certain nombre d'oppositions ruineuses : entre oralité et écriture, entre texte et contexte, etc.

9 Arrêtons-nous sur l'opposition convenue entre texte et contexte. Il est patent que le texte prend en charge jusqu'à un certain point son propre contexte, notamment via tout ce qu'on appelle le péritexte: préfaces, introductions, dédicaces, lettres d'accompagnement, correspondance, etc., ce qui conduit à se demander où commence au juste le texte et où il se termine. Descartes, par exemple, demandait expressément que les Méditations ne soient pas détachées des réponses aux objections. Considérer qu'elles constitueraient seules le véritable texte et que les objections et réponses n'en seraient que les parerga est donc faire violence au projet même de l'auteur. C'est en outre cette prise en compte des parerga qui conduit à mettre l'accent sur le contexte institutionnel, pour autant que, muni de son péritexte, tout texte apparaît comme un texte d'intervention ${ }^{3}$. C'est elle enfin qui permet une analyse fine des différences de statut entre les différentes œuvres, ressortissant généralement à différents genres, où se monnaie la présentation de la doctrine ${ }^{4}$.

10 On peut noter également que le texte se rapporte directement à son contexte, via un certain nombre de références, de dénominations (noms propres ou expressions définies), d'instanciations - contexte que pour partie il réorganise et reconstruit, donc contribue peu ou prou à transformer. Prenons pour exemple les nombreuses «Pensées » de Pascal où il est question de « l'Eglise » : on a là affaire à une sorte de nom propre se présentant comme l'instanciation au sein du texte d'une institution ou d'une autorité extérieure, instanciation qui permet au propos pascalien de s'articuler sur les enjeux théologiques et religieux de son temps (en vérité politico-religieux puisque Pascal réfléchit en l'occurrence sur la politique de l'Eglise), enjeux au sein desquels l'auteur entend se situer activement.

11 Autre exemple: les Dialogues sur la religion naturelle de Hume. Les positions qui s'y trouvent confrontées à travers la dispute sont non seulement nommées - ou plus exactement qualifiées - mais elles se trouvent du même coup instanciées. Qualifiées, tout d'abord: ce sont les protagonistes eux-mêmes qui baptisent leurs positions respectives, selon des noms de courants ou de doctrines qui se trouvent du même coup instrumentalisés dans le cadre de l'argumentation. On ne saurait sous-estimer l'importance de ces actes de nomination qui constituent de facto des arguments. Montrer, par exemple, que l'argument de l'adversaire fait le jeu de l'athéisme ou nous fait tomber dans la philosophie abstruse constitue autant de coups gagnants ${ }^{5}$.

12 Ces positions, disions-nous, ne sont pas seulement qualifiées mais instanciées : ces objets paradigmatiques que sont «le mysticisme», «l'anthropomorphisme», 
contribuent à montrer qu'un débat n'est jamais purement «théorique». Les protagonistes ne parlent pas seulement pour leur propre compte. Défendant des positions, ils parlent au nom d'autre chose qu'eux-mêmes. Leurs discours s'ordonnent à des instances, à des institutions qui sont censées faire autorité. «Religion positive » ou "théologie révélée », "religion naturelle, " «scepticisme outré»: telles sont les instances que les protagonistes font, en dernière analyse, jouer les unes sur les autres, ce qui permet de convoquer des doctrines en les investissant et en les désinvestissant tour à tour de leur autorité doctrinale. Encore ne faudrait-il pas limiter l'analyse aux seuls noms propres ou aux titres de doctrines: des expressions comme "la raison", " l'expérience ", « l'autorité », « l'évidence ", « la science ", « la piété » constituent elles aussi des instances, de quasi-personnages conceptuels dont la convocation discursive est censée produire des effets de validation, presque des témoignages et, pour finir, instancier les normes supra-discursives sans quoi aucune discussion ne pourrait faire autre chose qu'aligner des arguments face à d'autres arguments. De fait, sans la prise en compte de ces dimensions d'adresse et de positionnement, il n'est pas possible de prendre la mesure exacte de l'argumentation déployée par le texte. L'Analyse du Discours nous invite en ce sens à rompre avec une conception purement logicienne de l'argumentation sans revenir pour autant à une approche rhétorique restreinte. Le cas exemplaire des dialogues philosophiques montre que des argumentations également consistantes peuvent être confrontées, et que c'est cette confrontation qui permet de mettre au jour un certain nombre de présupposés et de partis pris pour partie indécidables (Hume), sauf à les soumettre à un principe de choix (Platon).

\section{Textualité et discursivité : une différence pour partie irréductible}

C'est donc, apparemment, de façon tout à fait naturelle que l'Analyse du Discours se trouve conduite à récuser toute différence de principe entre discours et textes, et à considérer que ces derniers relèvent de plein droit de ses méthodes. Mais on peut se demander si cette opposition entre approche discursive et approche immanentiste des textes ne constitue pas une alternative quelque peu forcée, faisant dans sa radicalité même l'impasse sur un possible troisième terme. Une chose est en effet de jeter un regard critique sur toute approche qui aborde le texte de façon purement immanentiste; une autre de soutenir que les textes sont, dans une large mesure, assimilables à des discours; une troisième serait de se demander si une telle assimilation est possible sans reste, autrement dit s'il n'existe pas des dimensions du texte qui sinon résistent du moins échappent à un type d'approche purement discursive. Resterait alors à déterminer le point à partir duquel d'autres méthodes d'approche, plus proprement textuelles, devraient en quelque sorte prendre le relais. Le point en question est, en l'occurrence, difficile à déterminer parce que, si l'on s'en tient aux contributions des auteurs qui ont donné toute sa consistance au programme d'une Analyse du Discours appliquée aux textes philosophiques ${ }^{6}$, la frontière entre le proprement discursif et le spécifiquement textuel apparaît difficile à assigner, tous deux considérant manifestement que l'Analyse du Discours peut accorder un rôle essentiel à l'analyse textuelle, sans qu'il soit requis de thématiser la différence entre discours et texte. 

du cadre théorique de l'Analyse du Discours, Maingueneau propose de distinguer ce qu'il appelle "scène englobante", "scène générique " et "scénographie " (2015: 81-91). Or, au moment de présenter ce troisième concept, il estime plus fécond de le diviser à son tour en "scénographie situationnelle " et "scénographie architectonique ». Via cette notion de "scénographie architectonique», il se propose de montrer en quoi « l'énonciation structure le déploiement de ses contenus » (82). Et d'étayer cette proposition sur une brève analyse comparative des démarches suivies respectivement par Spinoza et Descartes: tandis que chez Spinoza, les marques de l'énonciation sont le plus possible effacées au bénéfice de la mise au premier plan de l'architectonique, chez Descartes la « scénographie méditative » vient au premier plan «sans pour autant masquer la dimension architectonique, clairement perceptible dans le découpage en six méditations » (ibid.).

Mais comment s'enquérir de la dimension architectonique en question sans pratiquer d'abord une analyse textuelle des plus classiques? Quant au choix, par Descartes, de la scénographie méditative, il suppose, pour être avéré, l'analyse serrée d'un protocole d'écriture. Concernant enfin le concept même d'énonciation, il reste à décider s'il constitue une catégorie exclusivement discursive: la complexité des procédés énonciatifs dont usent les grands textes est souvent telle que seule une analyse textuelle minutieuse peut en rendre compte. Nous sommes donc face à un entrelacement $\mathrm{du}$ discursif et $\mathrm{du}$ textuel qui conduit peut-être à renverser la perspective : rien n'interdit certes à l'Analyse du Discours d'intégrer à son champ les ressources de l'analyse textuelle, mais on peut soutenir à l'inverse que c'est à l'analyse textuelle d'user des ressources de l'Analyse du Discours, comme l'une des diverses disciplines qu'elle a à sa disposition.

Si l'on se tourne à présent vers le travail de Frédéric Cossutta, autre grand représentant de l'Analyse du Discours appliquée aux textes philosophiques, on observe, dans le même esprit, que le couple catégorial d'origine hjelmslevienne dont il use volontiers en mettant en parallèle "schèmes doctrinaux » et "schèmes d'expression ${ }^{7}$, suppose lui aussi, pour être pleinement opératoire, d'être référé à des textes, qui constituent en vérité le seul lieu où se donne à voir l'élaboration des catégories à travers leur expression discursive. Certes, l'ambition de Cossutta est de ne pas se limiter au texte entendu stricto sensu mais, fidèle à un programme d'inspiration gueroultienne, de prendre en compte la doctrine ou le système dans son intégralité, les œuvres se présentant alors comme des expressions et donc des spécifications génériques de la doctrine considérée dans sa totalité. Mais même en admettant que cette catégorie de genre relève de plein droit de l'Analyse du Discours, il reste que la démonstration ne peut être complète qu'à la condition d'entrer dans le dispositif architectonique de l'œuvre, ce qui mobilise, de facto, les ressources de l'analyse textuelle. On peut certes réindexer toute l'entreprise à la seule notion de discours, le concept même d'« expression " étant sans doute choisi à dessein. Mais on peut à l'inverse contester cette subsomption du champ de l'analyse sous le seul concept de discours, refuser les équivoques du mot "discursif ». L'usage que fait l'Analyse du Discours de cet adjectif vient à la fois recouvrir et oblitérer la distinction philosophique canonique entre « discursif » et « intuitif». Affirmer que toute pensée prend nécessairement une forme discursive n'implique pas qu'elle puisse et doive être assimilée sans reste à un discours. A l'inverse, prendre en compte la dimension de l'écriture dans l'élaboration et la 
production de la pensée permet de montrer qu'elle comporte des éléments graphiques, topologiques, iconiques, et met en œuvre des schèmes dont l'analyse minutieuse permet d'éclairer ce qu'on appelle «intuition». Toutes raisons qui conduisent, méthodologiquement, à se soucier du fait que les «formes d'expression » en question, dès que l'on se situe au-delà des seules considérations génériques, sont d'abord et essentiellement des formes d'écriture.

Reste la question la plus délicate, celle du concept. Comme on le constate aisément, l'Analyse du Discours, même lorsqu'elle s'intéresse expressément au discours philosophique, ne fait guère de place à la question du concept. Non que la chose soit en droit impossible: les concepts que mobilise un auteur ne tombent pas du ciel, ils mènent eux aussi une sorte d'existence dans l'interdiscours ${ }^{8}$. Mais le travail de réélaboration, de rethématisation auquel les soumet un auteur n'est possible qu'au sein de cette "machine spéculative " que constitue le texte. Il est donc illusoire de croire qu'on aurait accès au système ou à la doctrine d'un auteur sans le travail patient de déchiffrement des textes où cette doctrine se trouve non seulement consignée mais pour une bonne part élaborée. On ne peut considérer les doctrines comme des objets immédiatement disponibles, que nous aurions en quelque sorte sous la main et qu'il suffirait de convoquer sans s'interroger sur les sources dont est issu le "savoir " que nous en avons. Or l'idée que nous nous faisons des "contenus doctrinaux » est, que nous le voulions ou non, empruntée à ce type de savoir qu'a accumulé une longue pratique du commentaire telle que la privilégie l'histoire de la philosophie. Il vient un moment où, pour régler finement le rapport qu'entretiennent formes du contenu et formes d'expression, s'impose le retour méticuleux au texte.

Il convient donc, pour délimiter le champ où l'Analyse du Discours est véritablement opératoire, de faire l'effort de thématiser et la différence entre texte et discours, et leur articulation ${ }^{9}$. Que tout texte comporte une dimension rhétorico-discursive, cela va de soi dans la mesure où il a un destinataire, vise un auditoire, se réfère à l'interdiscours, mentionne les instances discursives où se monnaient contradictoirement des formes complexes de véridiction et d'autorité, entendant produire dans le champ ainsi constitué des effets de clarification, de disqualification, de réorganisation. Il y a en ce sens une pragmatique textuelle, qui ne se réduit d'ailleurs pas aux seules dimensions éthiques, cathartiques ou protreptiques ${ }^{10}$ des textes philosophiques " classiques ».

Mais il n'en faut pas moins maintenir qu'un texte est plus et autre chose qu'un discours. Ou que le statut du texte, et plus particulièrement ce qui constitue proprement sa textualité, ne se laissent pas entièrement capturer par le concept de discours, quelle que soit l'extension que l'on entend donner à ce dernier. Le texte non seulement déploie mais en un sens constitue un espace spécifique où s'exprime une démarche intellectuelle, et où la pluralité des discours qui constitue l'interdiscours est susceptible de rejouer sur une scène rigoureusement circonscrite où ceux-ci se trouvent discriminés, confrontés, hiérarchisés. C'est cette spatialité propre au texte ${ }^{11}$ et la capacité intégrative qui lui est associée qui le rend irréductible à la relative linéarité du discours.

\section{Qu'est-ce qu'un texte?}

Posée dans sa généralité, une telle question exigerait que soit reconstituée l'histoire culturelle et intellectuelle d'un concept qui a connu bien des vicissitudes ${ }^{12}$. Dans la 
mesure où notre enquête porte plus particulièrement sur les textes philosophiques, nous nous contenterons de quelques indications.

\subsection{Oralité et écriture}

21 On est un peu surpris de la facilité avec laquelle les représentants d'un certain nombre des disciplines qui prennent pour objet la langue ou le discours dénient toute pertinence à la distinction entre oralité et écriture. On peut, si l'on veut, ignorer les critiques que Derrida a adressées à cet alignement de l'écriture sur la parole, on ne saurait pour autant faire passer à la trappe ce qui constitue la spécificité manifeste de l'écrit, ou plus exactement ses possibilités formelles, possibilités qu'il peut certes ne pas déployer (il est vrai que certaines expressions écrites ne sont guère que le simple réencodage de performances orales), mais dont il peut en revanche jouer pleinement, comme c'est le cas dans ces textes complexes que sont les œuvres, particulièrement celles des philosophes. Examinons quelques-unes des ces propriétés formelles.

Le texte s'adresse à un lecteur et non pas à un auditeur ${ }^{13}$. L'acte de lecture n'est donc pas assimilable à une relation d'écoute. Il se trouve que le destinataire-auditeur d'un échange conversationnel par exemple est en mesure de répondre immédiatement au discours qui lui est adressé. Le propre de l'écrit est à l'inverse de différer, de suspendre, voire d'abolir l'échange entre l'énonciateur et le lecteur. Un lecteur peut certes donner une réponse différée (dans le cadre d'une correspondance par exemple), mais il saute aux yeux qu'une simple correspondance écrite n'est pas assimilable à une conversation. De fait, ce qui caractérise l'écriture, c'est qu'au moment d'écrire, le destinataire est absent à l'énonciateur, tandis qu'au moment de lire l'auteur est absent au lecteur. Cette double absence modifie totalement les conditions, et de l'écriture, et de la réception. De fait, la plupart des textes sont destinés à un lectorat dont l'auteur n'a qu'une idée partielle, tandis que symétriquement le texte, lorsqu'il s'agit d'une œuvre publiée, échappe à son auteur et pour finir s'émancipe jusqu'à un certain point du contexte où il fut écrit ${ }^{14}$. Quant à la lecture, elle n'est en rien une sorte de réplique que le lecteur adresserait à l'auteur (d'où le paradoxe et pour partie l'illusion herméneutique consistant à faire du sens de l'œuvre la somme des lectures, voire des contresens effectués sur elle).

Certes, le discours lui-même ne saurait être identifié sans reste au type de parole qui circule dans l'échange conversationnel. Lui aussi s'adresse à un auditoire virtuel qui ne se confond pas avec l'auditeur réel. Son adresse est complexe, surdéterminée, et le jeu orchestré par le dialogue platonicien en est d'ailleurs une illustration familière. Mais cette complexité est démultipliée par l'écrit, ce dont la «forme dialogue » fournit précisément une preuve exemplaire.

\subsection{L'exemple du Dialogue}

24 Arrêtons-nous un instant sur le cas, tout à fait décisif, de cette "forme dialogue ». Maingueneau note la difficulté qu'ont les représentants de l'Analyse du Discours habitués à travailler dans le champ de l'analyse conversationnelle, à prendre en compte le dialogue ${ }^{15}$. Mais lui-même semble penser qu'une analyse recentrée effectivement sur le discours (qui est plus et autre chose qu'une simple parole) s'applique du même mouvement au dialogue oral et au dialogue écrit. Or le dialogue écrit n'est pas, il s'en 
faut de beaucoup, la simple transcription d'un dialogue soutenu oralement. Il se présente comme une œuvre littéraire autrement complexe. L'analyse effective d'un dialogue de Platon ou d'une œuvre comme les Dialogues sur la religion naturelle de Hume en donnent une illustration éclatante. Le (ou les) dialogue(s) ${ }^{16}$ s'y présente(nt) comme beaucoup plus qu'une simple discussion, c'est-à-dire un échange d'arguments : ils se présentent plus exactement comme un espace de confrontation des argumentations ${ }^{17}$. La «forme écrit " se révèle ici essentielle: sans elle, impossible de convoquer des argumentations complexes dans un même espace, de les faire jouer les unes sur les autres, d'en préciser certaines inflexions stratégiques via des didascalies et surtout, de dégager leurs aspects méta-argumentatifs ${ }^{18}$.

A quoi s'ajoute le fait qu'un dialogue écrit n'est jamais un pur dialogue. D'une manière générale, le propre de toute forme écrite est de pouvoir accueillir au sein d'une forme générique donnée bien d'autres formes, qui s'y trouvent enchâssées. Il est aisé de montrer, par exemple, que le dialogue - sous la plume de Platon - se présente comme une forme susceptible d'accueillir toutes les autres formes. On y a des parties dialoguées bien sûr ${ }^{19}$, mais aussi des récits, des dialogues rapportés, l'insertion de toutes sortes de discours suivis: tirades, éloges, apologies, prosopopées, allégories, mythes, etc. Toutes ces formes sont présentes, même à titre d'inserts, comme de possibles expansions d'une unique forme qui fournit à la scène discursive son niveau référentiel ultime : le dialogue.

Celui-ci apparaitrait donc comme la seule solution permettant d'accueillir mimétiquement, tout en les distanciant, les diverses scènes de discours où s'exhibe la parole intellectuelle au temps de Platon. Vaste forum d'écriture où vont pouvoir se trouver exhibés, soutenus, plaidés, confrontés tour à tour tous les genres de discours : rhétorique, éristique, entretien socratique, éloge funèbre, etc. Tous ces genres doivent être mimés ${ }^{20}$ pour être thématisés comme autant de pratiques possibles de discours. Le dialogue, loin de confronter seulement des thèses, des écrits, des objets de discours, met en présence des gestes, des pratiques discursives, avec leurs codes, leurs règles, leurs procédures : le dialogue constitue une scène d'emblée méta-discursive, où le sens, l'effet de vérité n'apparaît pas seulement lié à ce que les discours soutiennent ou énoncent mais tout autant à la façon dont ils l'énoncent et sont amenés, du même coup, à jouer différentiellement les uns sur les autres.

On pourrait donc considérer le dialogue comme le lieu où peuvent se rencontrer des pratiques ou des régimes de discours qui ne trouvent normalement pas à s'ajuster et qui doivent, nolens volens, inventer les conditions d'une possible transaction ou contribuer à exhiber la structure d'un différend. La difficulté, voire l'impossibilité de cet ajustement, l'incompatibilité des discours - donc à la fois des thèses et du mode de leur affirmation - peuvent donc être simultanément jouées et réfléchies.

\subsection{Spécificité de l'objet textuel}

29 Mais l'enjeu véritablement décisif consisterait à déterminer en quoi consiste la textualité même du texte ${ }^{21}$. Un texte doit être considéré, conformément à son étymologie d'ailleurs, comme un objet bi-dimensionnel. Certes, le processus de lecture est un processus nécessairement linéaire. Mais, outre que le texte est un objet offert en permanence à la relecture, ce qui le caractérise encore, comme l'a parfaitement vu Gilles Gaston Granger, c'est une forme de récurrence. Non seulement, plus encore que 
pour le discours, le texte est en mesure de multiplier les renvois à lui-même et de préesquisser, pour le lecteur, sa propre configuration mais surtout, son dispositif linéaire se trouve comme redoublé par le jeu des récurrences terminologiques ou argumentatives. La récurrence d'un concept ou d'une formule, la transformation rhétoriquement réglée d'une structure argumentative proposent au lecteur comme un second protocole de lecture qui vient doubler la lecture linéaire, ouvrant sur le texte des perspectives transversales. Lorsque telle page de Rousseau ou de Pascal tisse dans le texte le jeu de la récurrence d'une distinction cardinale (Lhomme 2014), le lecteur est incité à développer une sorte d'attention seconde, à pratiquer comme une lecture au deuxième degré que l'on pourrait dire «verticale " pour l'opposer à la linéarité du propos argumentatif de premier degré.

Le texte présente en effet des régularités, des reprises et des retours, des modulations ou des variations qui lui donnent un caractère non plus linéaire mais matriciel, lui conférant comme une deuxième dimension, à proprement parler une texture. Ce qui rend pertinentes les analogies que l'on est accoutumé de faire tant avec la composition musicale qu'avec la construction architecturale. Le texte présente un espace propre, et tout aussi nécessairement un tempo. C'est d'ailleurs à partir de ces considérations relatives à sa spatialité et son tempo que l'on peut comprendre en quoi le texte présente une dimension proprement pensante: dès lors que les chaînes d'argumentation ont atteint une complexité suffisante pour que leur seule lecture de proche en proche ne suffise pas à se les approprier. Le texte présente en ce sens une dimension opératoire et combinatoire, invitant le lecteur à réeffectuer les compositions d'arguments, à souligner au moins mentalement les concepts qui en constituent les nœuds, bref à faire preuve à son tour d'une forme d'imagination spéculative. On comprend donc que cette réhabilitation conjointe de la textualité et de la forme écrite conduise à s'intéresser moins à ce qui constitue l'événement discursif qu'à la manière dont, dans l'œuvre, une pensée cherche et trouve à s'inscrire pour déployer son inventivité propre : moins donc à la dimension de destination, de contextualisation ou d'énonciation qu'à cette dimension opérante et combinatoire qui ne s'ouvre et ne se déploie pleinement que dans l'écriture. Ce qui revient à poser en principe que les énoncés philosophiques n'ont pas seulement une signification et une portée qui tient à leur destination : il y a en toute pensée une dimension de figuration, de schématisation, voire de spatialisation (une dimension soit topologique, soit diagrammatique) dont l'analyse suppose d'accorder une sorte de primat méthodologique à l'écriture ou au texte par rapport au discours.

\section{Quel type d'analyse textuelle pour le texte philosophique?}

31 Nous proposons, pour finir, d'esquisser deux grandes voies d'analyse susceptibles de donner une véritable consistance au type d'analyse textuelle que nous semble requérir le texte philosophique ${ }^{22}$.

\subsection{Pour une approche stylistique des textes philosophiques}

Une première voie d'approche consisterait à redonner à la question du style toute sa pertinence en la liant à l'analyse méthodique des formes d'écriture. La catégorie de 
style présente en effet l'avantage d'attirer d'emblée l'attention sur ce qui fait la singularité du procès d'écriture, considérée à son tour comme le symptôme de ce qui fait la spécificité d'une forme de pensée. Elle donne ensuite quelque crédit à un certain type d'études comparatives, études qui n'ont donné lieu jusqu'ici qu'à des tentatives très inégalement réussies mais qui continuent de hanter plus ou moins obscurément la pratique empirique de l'histoire de la philosophie. Enfin, une stylistique du texte philosophique, attentive aux figures et aux schèmes - rhétoriques, argumentatifs, iconiques - qu'actualise le procès d'écriture, devrait offrir une voie d'accès privilégiée aux opérations à travers lesquelles se déploie et se constitue un procès de pensée et déboucher plus généralement sur une véritable poïétique de la pensée. Cette " théorie " $\mathrm{du}$ style, il faut, pour lui donner toute son ampleur dans le champ des études philosophiques, la référer aux deux auteurs qui ont su lui donner une véritable consistance, à savoir Leo Spitzer et Gilles Gaston Granger.

Dans ses célèbres Etudes de style (1970), Spitzer nous invite non seulement à privilégier les études empiriques mais à faire fructifier une méthode qui, partant du repérage différentiel des "traits de style ", s'efforce ensuite de repérer ceux qui présentent un caractère d'analogie pour essayer de dégager la " formule synthétique » ou "l'essence » d'un style, comme le dit Jean Starobinski dans sa préface (1970:20). On peut, certes, ne pas partager la théorie de l'expression dans laquelle s'inscrit originellement cette approche et, cherchant à articuler de façon plus «immanente » la catégorie de style elle-même en y voyant plutôt la forme de concaténation des éléments d'un discours ou d'un texte, refuser de référer directement l'expression à l'Erlebnis de l'auteur ou même à quelque etymon spirituel, préférant, en dernière analyse, mettre l'accent sur le travail de composition de l'œuvre plutôt que sur sa dimension « subjective ».

Encore faut-il souligner que Spitzer lui-même n'a cessé de s'éloigner de la valorisation post-diltheyenne de l'Erlebnis et de dépsychologiser sa propre démarche. Reste que, pour prendre toute son ampleur, ce remaniement critique exigeait d'aller au-delà d'une définition du "trait de style » comme simple écart ou comme déviation. De fait, dans un deuxième moment de sa réflexion, Spitzer a fini par préférer à la notion de variation ou d'écart différentiel celle de « détail » - ce type de singularité que le critique a pour vocation de repérer et d'élire en le considérant comme représentatif de la structure d'ensemble : c'est quand "le détail et le tout ont trouvé leur dénominateur commun » qu'ils "nous donnent la racine du texte» (op. cit.: 67), selon une relation d'entreexpression entre la partie et le tout qui exige, pour être pleinement maitrisée, de s'engager résolument dans le « cercle de la compréhension ».

Certes, la reprise spitzerienne des paradoxes du «cercle herméneutique » théorisé par Schleiermacher ne va pas sans quelques difficultés, le va et vient entre ce que Starobinski appelle «le détail ainsi éclairé » et le "tout pressenti » pouvant avoir quelque chose d'encore trop intuitif et donc d'aléatoire. Mais il suffit, pour y échapper, de faire un pas de plus et de considérer que ces traits de style, d'abord repérés comme de simples variations ou comme des écarts, en viennent à dessiner de véritables motifs qui singularisent de façon morphologique - et plus seulement différentielle - une œuvre déterminée. Motifs qui constituent autant de médiations déterminées entre «l'exégèse du détail » et la compréhension d'ensemble de l'œuvre. Reste que - et c'est la raison pour laquelle on parlera d'« études de style " plutôt que d'une véritable "stylistique » entendue comme discipline autonome -, il n'est pas sûr que ce type d'approche stylistique puisse jamais prendre la forme d'une «science en soi »: elle 
aurait plutôt le statut d'une critique mobilisant, au service d'une lecture toujours singulière, l'ensemble des ressources que lui offrent les diverses disciplines qui se partagent le champ des études textuelles : grammaire, linguistique, rhétorique, théorie de l'énonciation, sémiotique, théorie de l'argumentation, etc. Son apport théorique le plus spécifique consistant, par-delà le repérage des différences et des singularités qu'elle découvre sur ces divers plans, à fournir les matériaux d'une sorte de théorie des formes, entée sur la théorie classique des figures.

L'autre référence fondatrice en matière d'études de style, c'est bien sûr L'Essai d'une philosophie du style de Gilles Gaston Granger (1968). En s'efforçant de donner un fondement théorique plus consistant à la notion même de style, Granger commence par en généraliser considérablement l'acception, ce terme désignant désormais rien moins que le «processus d'intégration de l'individuel dans un processus concret qui est travail ». Une telle détermination conserve malheureusement un caractère quelque peu équivoque, dans la mesure où elle ne distingue pas suffisamment l'individuel - toujours menacé d'être restreint, dans la représentation commune, au champ de la personne particulière - et le singulier, catégorie permettant de penser de façon beaucoup plus générale l'ensemble des accidents et des singularités qui caractérisent ou affectent un procès, quels qu'en soient le domaine ou le support.

37 Mais ce qui retiendra plus particulièrement notre attention, c'est que Granger a eu l'audace de généraliser l'emploi de la catégorie de style jusqu'à la transférer dans le champ des théories formelles et plus particulièrement dans celui des mathématiques, montrant du même coup que la notion de style ne saurait, en droit, caractériser les seuls phénomènes d'expression mais qu'elle trouverait sa source dans certaines propriétés des théories formelles, considérées moins via leur réécriture, en droit intégralement formalisée, que dans leur procès d'élaboration, procès dont l'indétermination partielle ou la surdétermination expliquent précisément le caractère heuristique. Cessant donc de considérer le style comme une simple modalité de l'expression, Granger désigne plutôt à travers lui l'ensemble de ces singularités de l'expression qui résultent du choix d'un type déterminé de symbolisme. D'une manière générale, le choix d'un symbolisme déterminé comporte des redondances, des équivoques ou des surdéterminations qui en individualisent et en historicisent l'usage.

Mais le plus décisif tient à ce double fait que

- au plan des formes d'expression, toute écriture comme toute langue présente une "superposition de plusieurs niveaux de structures subordonnées ». Et c'est dans le jeu qui est susceptible de s'instituer entre ces divers niveaux que se produisent ces phénomènes de redondance non aléatoires qui sont source d'effets de sens surdéterminés,

- au plan plus proprement théorique et conceptuel, il est fréquent qu'un même problème apparaisse susceptible de plusieurs traitements, voire de plusieurs solutions non homogènes.

A côté de l'idée téléologique certes parfaitement efficiente selon laquelle il est toujours possible, en droit, de réduire cette pluralité des types ou des styles de traitement via une formalisation toujours plus poussée, il faut maintenir cette idée que la diversité des styles est coextensive à l'usage même de tout symbolisme. Le style apparaît ainsi « intimement lié à l'esprit des méthodes » (Chasles, cité par Granger, 22) et c'est en quoi il permet de concevoir des études comparatives, comme celle que Granger consacre à Descartes et Desargues - jetant du même coup un éclairage précieux sur la différence, 
en mathématiques comme en philosophie, des styles cartésien et pascalien. Car on voit mal comment une catégorie considérée comme parfaitement opératoire dans le champ des théories mathématiques ne présenterait pas un caractère heuristique au moins équivalent dans le champ de la conceptualité philosophique.

Pour résumer l'objet de cette stylistique renouvelée, exploitant le double apport de Spitzer et de Granger, je dirai que, pour s'engager dans une étude proprement stylistique des textes philosophiques, il suffit de considérer que ceux-ci comportent généralement des schèmes, des motifs ou des formules, à caractère introductif ou générateur, qui munissent le lecteur de ce que j'appellerais volontiers des structures d'attente. De fait, certains de ces motifs constituent autant de singularités, soulignées, signalées par le texte, selon une forme de redondance qui peut se transformer en surdétermination. Non seulement le texte s'annonce, s'anticipe constamment, mais il munit le lecteur de formules remarquables, qui constituent autant de clefs de lecture. Si un texte ne se lit jamais de façon strictement linéaire, c'est parce que, sur cette structure linéaire, viennent se surimposer de multiples dimensions dont l'écriture joue, tissant un réseau beaucoup plus complexe d'arguments. Ce qui implique de doubler la lecture strictement logico-argumentative d'une lecture ouverte à d'autres dispositifs : rhétorique, topique, analogique, métaphorique, etc., de façon à repérer non seulement l'articulation des concepts, le réseau des métaphores filées et des analogies, mais la façon même dont le texte configure, dans le procès de son écriture, une partie des opérations intellectuelles qu'il invite son lecteur à effectuer.

Dans ce travail de figuration et de schématisation, certaines formules, par le jeu de leur récurrence et plus encore par celui de leur reformulation et de leurs transformations occupent une fonction stratégique. Elles jouent en effet un rôle inducteur dans la composition du texte, au point que celui-ci se présente parfois comme le déploiement méthodique d'une formule proposée dès l'ouverture $d u t^{2} \sec ^{23}$, formule que sa récurrence transforme en une véritable matrice générative. Ce type de rhétorique, spécifiquement philosophique, ne se présente donc pas comme une écriture "seconde " qui viendrait habiller des contenus de pensée conçus sans elle. Elle se présente très souvent comme le déploiement d'une formule conceptuelle elle-même structurée par une grammaire spécifique.

Pour achever de se convaincre de cette sorte d'équivalence entre rhétorique formulaire et écriture du concept, il faut bien sûr lever l'obstacle d'une théorie de la métaphore qui exclut systématiquement certaines figures, ne considérant que celles qui, de près ou de loin, s'apparentent à la métaphore au sens restreint ${ }^{24}$. L'analyse minutieuse des formules argumentatives dont usent un certain nombre de philosophes suppose en effet de faire venir au premier plan ce qu'on appelle "figures de construction »: parallélismes, chiasmes, etc., dont on peut montrer qu'elles sont en vérité de véritables opérateurs argumentatifs ${ }^{25}$. On peut montrer qu'en ce sens, l'écriture formulaire, loin d'être un jeu purement rhétorique, fournit à un grand nombre d'auteurs une véritable matrice de pensée (Lhomme 2014).

\subsection{La poïétique du texte : des formes d'écriture aux figures de pensée}

C'est la prise en compte de ces opérateurs qui permet de comprendre ce que nous proposons d'appeler la dimension poïétique du texte ${ }^{26}$. La production du sens, en effet, 
n'est pas assimilable à un simple "effet de texte ». Elle est immanente à la textualité même, dans la mesure où la composition du texte est en tant que telle indissociable d'une stratégie intellectuelle, autrement dit que le «travail de l'œuvre » constitue, en soi, un véritable ars inveniendi. Il ne suffit pas, en ce sens, de décrire ni même d'analyser la rhétorique rousseauiste : il faut déterminer du même mouvement ce qu'elle nous dit précisément de l'art de penser propre à Rousseau. Et donc de sa pensée elle-même, en tant qu'elle fomente, compute, littéralement produit le texte (Lhomme 2005). Car la pensée à l'œuvre dans le texte est loin de se réduire à la présentation linéaire d'une argumentation en forme. Le propre du texte est de croiser et de combiner de multiples lignes d'argumentation, qu'il lui faut ordonner, hiérarchiser, sur lesquels il est conduit à proposer des focalisations alternées, variant les types de preuve et parfois les styles démonstratifs.

Si donc l'on veut comprendre comment la pensée, véritablement, se produit et même se pense, il faut accepter de descendre dans le laboratoire que constitue le texte, autrement dit examiner les procédures et les schèmes sous-jacents à la formulation d'une pensée textuellement articulée. Il faut cesser de croire qu'écrire serait simplement donner sa juste formulation à une pensée toute faite, en quelque sorte purement méditante : écrire n'est pas autre chose que le travail même de formulation, donc d'effectuation d'une pensée.

On comprend donc que seule une étude attentive à la textualité permet de repérer, à même les faits d'écriture, les formes de réarticulation rhétorico-stylistique (ce que nous avons proposé d'appeler l'écriture formulaire) et logico-syntaxique, où se jouent de véritables opérations de pensée, et où affleurent des dimensions trop négligées par les approches spéculatives classiques ordonnées au seul concept: dimensions diagrammatique, topologique ${ }^{27}$, analogique et plus particulièrement schématisante. De fait, seule une théorie renouvelée du schématisme permettrait de réconcilier les dimensions simultanément discursive et intuitive qui constituent l'acte même de penser et d'en élucider simultanément la dimension imaginative et poïétique ${ }^{28}$. Tel serait le type de complétude propre à un authentique art de penser.

Mais pour comprendre pleinement la place que tiennent les faits d'écriture dans le travail textuel de production des concepts, il faut lever un ultime obstacle, tenant à la conception que l'on se fait des rapports entre le logique et le conceptuel, rapports dont les logiciens nous fournissent une version trop pauvre pour qu'elle soit véritablement opératoire. Pour comprendre comment des concepts peuvent se trouver textuellement configurés, il faut travailler à se doter d'une autre logique du concept, en se tournant vers les riche matériaux que nous fournissent des auteurs comme Cassirer (1977), opposant concepts génériques et concepts construits, ou Cavaillès (1960), opposant à l'opération logique classique de la généralisation-idéalisation le couple, fondé sur l'analyse serrée de la pensée mathématique, du paradigme et de la thématisation, seul ce dernier concept permettant de comprendre en quoi le dégagement des formes est le support de la création d'un nouvel objet, point de départ, à son tour, du dégagement de nouvelles formes. Plus généralement, l'examen, à partir des analyses proposées par Husserl (1965), du procès de nominalisation sous-jacent à la pensée conceptualisante, montre comment de simples propriétés peuvent se trouver portées au concept par un procès d'abstraction qui n'a plus rien à voir avec la généralisation aristotélicienne mais qui, en jouant par exemple sur les paronymes, permet de donner un sens inédit à de simples différences grammaticales: c'est ainsi qu'en thématisant la différence entre 
l'« existence » et l'« existant », Levinas (1990) parvient à proposer une ontologie qui, prenant son inspiration dans Etre et temps de Heidegger, en reformule radicalement les prémisses et en tire des conséquences éthiques insoupçonnées.

C'est ce concept parfaitement opératoire de thématisation qui permet de comprendre qu'un concept n'existe pas « en soi », qu'il n'existe pas non plus dans la pensée, comme un pur objet mental. D'un côté il se présente comme un index d'opérations en droit indéfiniment ré-effectuables, de l'autre il a son lieu dans la langue ou plus précisément dans l'interdiscours, où ces opérations thématisantes et re-thématisantes se trouvent en permanence ré-effectuées. En vérité, les concepts n'existent, c'est-à-dire ne s'actualisent, qu'à travers le travail d'appropriation, de reprise, de ré-effectuation qu'un auteur effectue à partir de discours transmis par une tradition expressément ou implicitement ré-interprétative, déposés aussi dans une archive ou diffusés dans la culture commune - travail de réeffectuation-réélaboration que l'auteur confie en quelque sorte au texte via son procès d'écriture. Car c'est au sein d'une œuvre, dans le laboratoire que constitue le texte philosophique, qu'un véritable et rigoureux travail de re-thématisation est possible. Encore faut-il prendre la peine d'en étudier les modalités et les formes. Et, pour en prendre l'exacte mesure, doubler l'indispensable analyse intra-textuelle d'études comparatives. Car il y a des styles de thématisation différents, chaque auteur explorant chaque fois un registre de possibilités différentes, en fonction d'un certain nombre de choix grammaticaux, sémantiques, logiques, discursifs, métadiscursifs. Tel serait le programme d'une approche stylistique des textes philosophiques.

\section{Conclusion}

Comme on voit, démêler les rapports entre Analyse du Discours et Analyse textuelle n'est pas chose simple. Plutôt que de les opposer, il faut les distinguer et pour cela déterminer leurs limites respectives, opération requise pour penser leur éventuelle complémentarité. Que les textes se présentent aussi comme des discours, je l'ai montré, et cela suffit à valider leur approche en recourant aux instruments de l'Analyse du Discours. Mais les textes, comme je l'ai montré également, ne sont pas entièrement assimilables à des discours. Ils présentent des dimensions que l'on peut certes intégrer à une Analyse du Discours conçue de façon extensive ${ }^{29}$ mais qu'il me paraît plus opératoire et plus pertinent de considérer comme relevant d'une approche spécifique ${ }^{30}$, que l'on préférera qualifier globalement d'Analyse textuelle, l'approche de type stylistique que j'ai proposée n'en étant qu'une composante. L'Analyse textuelle telle que je la conçois se présenterait en effet moins comme une discipline spécifique que comme une activité critique, mobilisant d'autres disciplines théoriquement constituées, auxquelles elle peut apporter de nouveaux matériaux, voire de nouveaux outils, mais dont l'élaboration suppose toujours une relation serrée à des textes singuliers. Mais elle ne peut, inversement, échapper à une approche « impressionniste » des textes comme aux facilités herméneutiques du « contresens fécond » qu'en mobilisant les ressources de ces disciplines constituées que sont la rhétorique, la linguistique, la grammaire, la théorie de l'argumentation et, bien sûr, l'Analyse du Discours. 


\section{BIBLIOGRAPHIE}

Achard-Bayle, Guy. 2006. «Textes/discours et co(n)textes, entretiens avec Jean-Michel Adam, Bernard Combettes, Dominique Maingueneau, Sophie Moirand », Pratiques 129-130, 20-49 Achard-Bayle, Guy. 2014. "Texte et discours se comprennent-ils? ", Monte, Michèle \& Gilles Philippe (éds). Genres et textes. Déterminations, évolutions, confrontations (Lyon : PUL), 23-38 Adam, Jean-Michel. 1999. Linguistique textuelle. Des genres de discours aux textes (Paris : Nathan) Adam, Jean-Michel. 2011. La linguistique textuelle (3ème éd.) (Paris : Colin)

Adam, Jean-Michel. 2014. « Le paradigme du texte : regard rétrospectif et perspectives pour les sciences des textes », Monte, Michèle \& Gilles Philippe (éds). Genres et textes. Déterminations, évolutions, confrontations (Lyon : PUL), 297-323

Adam, Jean-Michel. 2015. « L'analyse textuelle des discours. Entre linguistique textuelle et Analyse du Discours », Soulages Jean-Claude (éd.). L'analyse de discours. Sa place dans les sciences du langage et de la communication. Hommage à Patrick Charaudeau (Rennes : P. U. de Rennes) Benveniste, Emile. 2012. Dernières leçons (Paris : EHESS, Gallimard \& Seuil) Bonhomme, Marc, 2005. Pragmatique des figures du discours (Paris : Champion) Cassirer, Ernst. 1977 [1910]. Substance et fonction, éléments pour une théorie du concept (Paris : Minuit)

Cavaillès, Jean. 1960 [1947]. Sur la logique et la théorie de la science (Paris : PUF)

Cossutta, Frédéric. 1989. Eléments pour la lecture des textes philosophiques (Paris : Bordas)

Cossutta Frédéric. 1995. « Pour une Analyse du Discours philosophique », Langages 119, «L'Analyse du Discours philosophique », 12-39

Cossutta, Frédéric (éd.). 1996. Descartes et l'argumentation philosophique (Paris : PUF)

Galay, Jean-Louis. 1977. Philosophie et invention textuelle (Paris : Klincksieck)

Gardes Tamine Joëlle, 2011. Pour une nouvelle théorie des figures (Paris : PUF)

Gaston Granger, Gilles. 1968. Essai d'une philosophie du style (Paris : Colin)

Husserl, Edmund. 1965 [1929]. Logique formelle et Logique transcendantale (Paris : PUF)

Jacques, Francis. 1987. « Le moment du texte », Greisch, Jean (éd.) Le texte comme objet philosophique (Paris : Beauchesne), 15-85

Jacques, Francis. 2007. L'arbre du texte et ses possibles (Paris : Vrin)

Lhomme, Alain. 1998. « Formuler l'informulable, analyse d'un paradoxe pragmatique », Cossutta, Frédéric (éd). Lire Bergson (Paris : PUF), 101-138

Lhomme, Alain. 2001. « Le fils d'Hermès », Cossutta, Frédéric \& Michel Narcy (éds). La forme dialogue chez Platon (Grenoble : Jérôme Millon), 155-187

Lhomme, Alain. 2004. «L'éducation de Pamphile ou le théâtre de la raison sceptique », Cossutta, Frédéric (éd). Le dialogue : introduction à un genre philosophique (Lille : Septentrion), 207-235

Lhomme, Alain. 2005. « Entre concept et métaphore : existe-t-il une écrire spécifiquement philosophique? », Rue Descartes 50, « L'écriture des philosophes », 59-73 
Lhomme, Alain. 2014. « Formules philosophiques et écriture formulaire », Cossutta, Frédéric \& Francine Cicurel (éds). Les formules philosophiques (Limoges : Lambert-Lucas), 25-52

[à paraître :] Lhomme, Alain. 2019. " Thématisation et reconfiguration textuelle des concepts ", Cossutta Frédéric (éd.). La fabrique discursive des concepts philosophiques (Limoges : Lambert-Lucas)

Maingueneau, Dominique. 2015. La philosophie comme institution discursive (Limoges : LambertLucas)

Perelman Chaïm \& Lucie Olbrechts-Tyteka. 1970. Traité de l'argumentation (Bruxelles : Editions de l'Université de Bruxelles)

Ricœur Paul. 1986. Du texte à l'action. Essais d'herméneutique II (Paris : Seuil)

Spitzer, Leo. 1970. Etudes de style (Paris : Gallimard)

Steiner, Georges. 2011. Poésie de la pensée (Paris : Gallimard)

\section{NOTES}

1. Qu'on songe au Discours sur la condition des grands, de Pascal.

2. Un exemple très remarquable en est fourni par l'analyse que Maingueneau propose de la seconde Provinciale de Pascal (2015 : 169-178).

3. Comme en témoigne, dans les Méditations, la lettre adressée aux Docteurs de Sorbonne.

4. C'est le type d'analyse développé par Fréderic Cossutta (1996), notamment à partir du corpus cartésien. Cf. "Argumentation, ordre des raisons et mode d'exposition dans l'œuvre cartésienne » (en particulier 164-181).

5. De même, se faire taxer de mysticisme ou d'anthropomorphisme, de sceptique outré ou d'athée, constitue autant de « coups argumentatifs ».

6. Cf. Cossutta 1989, 1995, 1996 et Maingueneau 2015.

7. Cossutta parle aussi volontiers de «formes du contenu doctrinal» (voire de «contenus doctrinaux ») et de « modes d'expression » $(1996: 164)$.

8. On le montrerait aisément pour des notions comme celles de « religion naturelle » ou de « loi naturelle ». Mais cela vaut, en réalité, pour tout concept.

9. Le type d'articulation que nous proposons entre texte et discours recoupe pour une grande part les analyses de Jean-Michel Adam, telles que les restitue en particulier Guy Achard-Bayle dans la longue étude qu'il a consacrée à ses travaux, étude joliment titrée : «texte et discours se comprennent-ils ?». Interrogation qui revient à se demander « d'une part [...] si et sans quelle mesure l'un est inclus dans l'autre ; d'autre part, si et jusqu'à quel point ils sont compatibles » (Achard-Bayle 2014). Cf. aussi Achard-Bayle 2006 et Adam 2011, 2014 et 2015.

10. Le terme réfère à un " discours pour exhorter ", destiné à être lu, mais également écrit sur un mode oratoire (Wikipedia /NdlR).

11. Maingueneau parle significativement de « déploiement du volume textuel » $2015: 91)$.

12. Jean-Michel Adam a fourni à plusieurs reprises des éléments pour une telle histoire. Cf. notamment Adam (1999).

13. Je restitue ici, en les résumant, les analyses proposées par Ricœur dans la première partie de son célèbre article Qu'est-ce qu'un texte? (1986: 137-142), sans reprendre pour autant à mon compte les développements qui suivent concernant les notions d'explication et d'interprétation. Pour une réflexion sur la spécificité de l'écriture par rapport à la langue, cf. Benveniste (2012 : chapitre 2).

14. Ce qui caractérise en outre certains textes, c'est que l'auteur, dans le geste même de l'écriture, s'en est intentionnellement absenté. C'est particulièrement le cas pour les Dialogues de 
Platon mais c'est tout aussi frappant en ce qui concerne les Dialogues sur la religion naturelle de Hume.

15. « $\mathrm{Au}$ motif que ce ne sont pas des interactions authentiques mais des textes conçus par un auteur» (op. cit., p. 15).

16. Le pluriel, dans le cas de Hume, n'est nullement anodin.

17. Pour une analyse plus fouillée de la structure argumentative et des aspects métaargumentatifs des Dialogues sur la religion naturelle, cf. Lhomme (2004).

18. Dimension méta-argumentative qui ne concerne pas seulement la forme dialogue. On semble fondé à considérer que le propre d'un texte philosophique est de déployer une forme de scénographie argumentative où des arguments se trouvent non seulement exposés, légitimés ou délégitimés, distanciés ou rethématisés, mais inscrits au sein d'un nouvel espace textuel fonctionnant de facto comme un dispositif méta-argumentatif. Type de redéploiement que l'on peut certes trouver aussi partiellement à l'œuvre dans certains textes littéraires, juridiques, scientifiques, etc. (ce qui explique par exemple que la distinction texte littéraire versus texte philosophique soit parfois difficile à assigner). Le texte philosophique se caractériserait alors par le fait que le type de redéploiement argumentatif qu'il propose s'accompagne d'un effort de rethématisation des concepts impliqués dans les arguments.

19. Encore faudrait-il distinguer plus finement divers types d'échange dialogaux, tous ne relevant pas, loin de là, de l'ascétique entretien questions-réponses, dont les discussions éristiques et socratiques avaient fixé le canon.

20. Le dialogue est une scène : il est une mimesis d'entretiens, mais une mimesis distanciée, une mimesis corrigée, diégétiquement remaniée, déplacée. Voir Lhomme (2001).

21. Pour une réflexion systématique sur le sujet, cf. Francis Jacques $(1987,2007)$.

22. Nous ne prétendons bien sûr pas esquisser une théorie générale de l'analyse textuelle du type de celle que Jean-Michel Adam développe depuis près de trente ans sur le fondement d'une discipline déterminée : la linguistique textuelle (2011). Le type d'articulation que nous proposons entre Analyse du Discours et Analyse textuelle recoupe certes à bien des égards ses propres conclusions, mais en limitant la présente discussion au seul texte philosophique, nous donnons à dessein à l'expression " analyse textuelle " son sens obvie, celui qui prévaut dans ces « champs pratiques de l'explication de texte et de la stylistique » par rapport auxquels, selon Adam, la linguistique textuelle "doit se situer» (tout en les tenant épistémologiquement à distance : Adam 1999: 19 et $4 \mathrm{e}$ de couverture).

Nous réservons pour un autre travail l'examen du type de solution que propose Adam pour résoudre ce type de problème, via la formule syncrétique qui a désormais sa faveur, celle d'une « Analyse textuelle des Discours» $(2011,2015)$.

23. C'est le cas dans un célèbre texte de Bergson : "Le possible et le réel » (La pensés et le mouvant, Paris : PUF, 1978 [1938], cf. Lhomme 1998).

24. Il ne s'agit pas non plus, jouant unilatéralement la textualité contre l'oralité, de privilégier le seul couple texte-intertexte au nom d'une métaphore du texte généralisé et de résorber le travail de reprise sous-jacent au procès d'écriture dans une sorte de jeu de renvoi indéfini. Selon une lecture outrageusement simplifiée de l'entreprise derridienne, il pourrait sembler en effet que tout texte renvoie à un texte qui renvoie lui-même à un texte, et ainsi indéfiniment. Ce serait oublier l'autre dimension de la pensée à l'œuvre dans l'écriture : sa dimension schématisante et, concernant le texte philosophique, reconceptualisante.

25. La reconsidération du rôle proprement argumentatif des figures s'effectue dès la publication du Traité de l'argumentation de Perelman et Olbrechts-Tyteka (1970 : 225). Cf. également, sur le même sujet, dans une orientation plus expressément pragmatique, Bonhomme (2005 : 178-194), et surtout Joëlle Gardes Tamine (2011 : 158-160).

26. J'emprunte ce terme à Jean-Louis Galay, qui a cherché, dans son livre Philosophie et invention textuelle (1977), à en exploiter les ressources pour l'analyse des textes philosophiques. Mais la 
promotion de ce terme dans le champ esthético-critique remonte sans doute à Valéry (« Discours sur l'esthétique », Euvres 1, Paris, Gallimard 1957). Dans le même esprit, on peut mentionner le livre de Georges Steiner, Poésie de la pensée, où est évoquée « la pression formatrice incessante des formes du discours, du style, sur les programmes philosophiques et métaphysiques » (2011:15).

27. Dimension que nous avons essayé de mettre plus particulièrement en évidence via l'analyse d'une figure de rhétorique qui a connu, philosophiquement, une certaine fortune intellectuelle, celle du renversement. On peut bien sûr n'y voir qu'une métaphore spatiale qu'il faudrait soit récuser, soit traiter de façon symptomale : cela n'empêche nullement ceux qui critiquent son emploi, d'y recourir à l'occasion dans leur propre stratégie argumentative, reconnaissant par là qu'elle constitue en vérité un opérateur intellectuel, très exactement un schème topologique permettant d'esquisser au moins le graphe d'une argumentation (Lhomme 2014).

28. Qu'un certain type de schématisme, de type topologique notamment, soit sous-jacent à certaines formes d'imagination spéculative, j'ai essayé de le montrer (en 2014) via l'examen de deux démonstrations proposées respectivement par Sartre, dans L'Etre et le néant, et Levinas, dans Totalité et infini.

29. Cf. Cossutta 1989, 1995, 1996. Maingueneau 2015.

30. Rejoignant sur ce point la position soutenue par Jean-Michel Adam : «je défends l'idée de la nécessité, au sein du champ interdisciplinaire de l'analyse du discours, d'une théorie du texte » (Achard-Bayle 2006, p. 32).

\section{RÉSUMÉS}

Pour des raisons méthodologiques, l'Analyse du Discours est conduite à entrer dans un rapport polémique aux problématiques du texte, qui considèrent celui-ci avant tout comme un système signifiant et qui méconnaissent du même coup le caractère opératoire de catégories comme celles d'énonciation, de scénographie ou d'institution discursive. Tout en prenant acte de ce qu'apporte l'application aux textes des catégorise de l'Analyse du Discours, le présent article entend montrer quelles sont en revanche les dimensions du texte qui échappent à une approche proprement discursive, soulignant la résistance que le texte oppose à son alignement sur le seul discours. Il est possible de montrer à partir de là comment une analyse proprement textuelle est amenée à prendre en charge les fonctions méta-argumentatives et conceptuellement thématisantes que remplissent les textes philosophiques, tout en esquissant, pour en analyser finement les mécanismes, les voies d'une stylistique renouvelée.

For methodological reasons, discourse analysis tends to challenge text-focused approaches. The latter consider text as a system of meaning and therefore disregard the efficiency of categories such as enunciation, scenography or discursive institution. While taking note of the contribution of the categories of discourse analysis in the study of texts, this article places emphasis on the dimensions of texts that cannot be accounted for within discourse-focused approaches. It claims that a text resists its alignment with discourse. On this basis, one can demonstrate how a specifically textual-oriented analysis is able to take into account the meta-argumentative and conceptual functions that philosophical texts perform. Finally, this article sketches out the conditions of a new stylistics, which proves necessary to analyze in finite detail these various functions. 
INDEX

Keywords : Discourse Analysis, philosophical textuality, poïetics, stylistics, textual analysis Mots-clés : Analyse du Discours, analyse textuelle, poïétique, stylistique, textualité philosophique

\section{AUTEUR}

ALAIN LHOMME

Université Lille 3 